\title{
ECONOMIC ANALYSIS OF RIDE-SOURCING MARKETS
}

\author{
Liteng Zha ${ }^{\mathrm{a}}$, Yafeng Yin ${ }^{\mathrm{a}}$ * and Hai Yang ${ }^{\mathrm{b}}$ \\ ${ }^{a}$ Department of Civil and Coastal Engineering, University of Florida, 365 Weil Hall, \\ Gainesville, FL, United States \\ ${ }^{b}$ Department of Civil and Environmental Engineering, The Hong Kong University of Science \\ and Technology, Clear Water Bay, Kowloon, Hong Kong, PR China
}

\begin{abstract}
Ride-sourcing refers to an emerging urban mobility service that private car owners drive their own vehicles to provide for-hire rides. This paper analyzes the ride-sourcing market using an aggregate model where the matchings between customers and drivers are captured by an exogenous matching function. It is found that without any regulatory intervention a monopoly ride-sourcing platform will maximize the joint profit with its drivers. On the other hand, the firstbest solution is not sustainable when the matching function exhibits increasing returns to scale and the cost function of the platform is subject to economies of scale. Regardless of the examined market scenarios, the average waiting time of customers is proportional to the average searching time of drivers. We establish conditions for regulators to solely regulate the commission charged by the platform to guarantee the second best. We further investigate the competition of ride-sourcing platforms and find that competition does not necessarily lower the price level or improve social welfare. In the latter case, regulators may rather encourage the merger of the platforms and regulate them directly as a monopolist.
\end{abstract}

Keywords: Ride-sourcing, regulation, platform competition, matching friction

* Corresponding author. Tel.: 352-294-7805; Email: yafeng@ufl.edu 


\section{Introduction}

The number of smart mobile devices in the world has been rising steadily and a study suggests that nearly two-thirds of Americans now own at least one such device (Pew Research Center, 2015). These devices retrieve users' geolocations, enable ubiquitous communications and allow instant peer-to-peer interaction, giving rise to a new class of firms - on-demand companies - which aim to effectively bring together consumers and suppliers of resources (e.g., houses and parking spaces) and services (e.g., home cleaning and computer programming) with very low transaction costs. These companies are shaking up their industries and reshaping our daily lives.

As a typical example of on-demand economy, ride-sourcing companies such as Uber and Lyft are transforming the way we travel in cities. The companies provide ride-hailing apps and online platforms that intelligently source participating drivers to riders. A rider can monitor in real time the location of the coming vehicle and receive notification when it arrives. These apps are free to use but usually a commission is charged for each transaction/ride (15-20\% of the fare paid by the rider). Thanks to their convenience and competitive prices, ride-sourcing services have successfully attracted many riders, eroding the traditional taxi market.

Several terms exist for describing services provided by Uber-like companies, such as ridesharing, for-profit ridesharing, on-demand ridesharing, dynamic ridesharing (Anderson, 2014; Rayle et al., 2014). Conventionally, ridesharing is not for profit. In contrast, the provision of Uber-like services is largely driven by financial motive. Therefore, the California Public Utilities Committee proposed to use "Transportation Network Companies" to categorize Uberlike companies. Considering these companies are essentially sourcing a ride from a driver pool, Rayle et al. (2014) suggested the term "ride-sourcing" for such services. It is worth mentioning that major ride-sourcing companies also allow ride-splitting on their platforms to encourage multiple people to share a ride, e.g., UberPOOL, which may further blur the line between ridesharing and ride-sourcing. However, we choose to use "ride-sourcing" in this paper to highlight its distinctive feature, i.e., private car owners drive their own vehicles to provide taxilike services for profit.

Since their advent in 2009, ride-sourcing companies have enjoyed huge success, but also created many controversies. Because the regular taxi market is usually regulated in terms of price, entry and service quality while comparatively fewer regulatory requirements have been imposed on ride-sourcing companies (Frankena and Pautler, 1986; CPUC, 2013; CPUC., 2014), unfair competition is argued particularly by professional cab drivers and their employers, who have organized strikes and filed lawsuits around the world. Ride-sourcing companies have also brought headache to government officials and legislators. While many are still wondering what to do, some have decided to ban them or treat these services as illegal; others embrace them as a new type of transit provider or have passed ride-sourcing laws and regulations. Although these laws and regulations have some differences, they all essentially codify the insurance coverage, driver background check, and inspection protocols that ride-sourcing companies already have in place (Shaheen, 2014). There is no intervention on service fares or the size of the affiliated fleet (or the number of vehicles in service).

The success of ride-sourcing platforms has cast doubts on the regulation of the taxi industry that is often criticized for limited supply (Badger, 2014). It challenges some of the premises for the traditional taxi regulation by significantly reducing information imperfection (Frankena and Pautler, 1986; Cairns and Liston-Heyes, 1996; ECMT, 2007). Features such as real-time driverrider matching and mutual rating (the reputation system) offered by the platforms enhance the 
interaction between customers and drivers. Also, all trip-related fares are processed by built-in pricing and payment functions, which eliminate the chaos caused by soliciting and bargaining that often emerge after a taxi market is deregulated (ECMT, 2007). Last but not the least, some speculate that the ride-sourcing market is self-regulatory because the competition among multiple ride-souring platforms will lower the prices and reduce the market power of a particular predominant platform (Koopman et al., 2015). Should we simply deregulate the taxi industry and leave ride-sourcing companies alone, and then let the market decide the winners? What are the implications and welfare impacts of such a deregulation? Should we conduct a systematic reform of regulation of the ride-for-hire market? Presumably the reform needs to be tailored to specific cities considering their demographics, mobility options, patterns, and culture. Is there a unified theoretical framework to guide such a reform? Many such questions remain unanswered.

To help solve the puzzles and controversies associated with ride-sourcing companies, this paper makes a preliminary attempt to provide a quantitative analysis on the market structure of ride-scouring services and explores effective regulation policies. In this paper, we consider hypothetical situations when ride-sourcing companies become self-sustainable and dominate the ride-for-hire market. We are subsequently interested in knowing whether and how to regulate the ride-sourcing market. Following Yang and Yang (2011), we develop an aggregate model with a Cobb-Douglas matching function to examine different market scenarios, properties and economic outcomes of a hypothetical ride-sourcing market with a single platform. In view of the potential market distortion, we investigate effective regulation policies that require minimal regulatory variables. The analysis is further extended to consider a duopoly market to examine the effects of platform competition. Analyzing the tradeoff in the pricing formula under the Nash equilibrium, we observe that competition does not necessarily lower the price level. Via a numerical analysis, we explore the conditions where competition is socially inefficient and a regulated monopoly market can be more efficient than a regulated duopoly one.

The paper is organized as follows. The basic aggregate model for a hypothetical ridesourcing market along with some comparative statics is presented in the second section. The third section explores the pricing structure, solution properties of the single platform across different market scenarios as well as the regulation policies. In the fourth section, the discussion is extended to a duopoly market with a particular focus on the changes in price and social welfare. The paper then concludes with a summary of research findings and policy implications as well as a discussion of future research directions.

\section{Basic Model}

This section introduces an aggregate model that captures a hypothetical ride-sourcing market with a focus on the matchings between customers and drivers. The model is established by extending the work of Yang and Yang (2011). We firstly assume a hypothetical ride-sourcing market with a single platform, a group of customers and a group of affiliated drivers. The ridesourcing platform serves as an intermediary that matches customers with potential drivers. The platform decides the fare paid by a customer, i.e., $F$, and charges a commission, i.e., $P$, from the payment by the customer at each transaction; the driver receives the remaining payment, i.e., $F-P$. It is assumed that the hypothetical ride-sourcing market is mature such that the platform will gain profit from providing the services. A few other things are worth noting here: 1) unlike ride-sourcing platforms, e-hailing platforms for traditional regulated taxi market do not have price-setting power (He and Shen, 2015); 2) although in practice ride-sourcing platforms charge 
commission as a percentage of the fare, such a distinction is immaterial in the context of this paper; 3 ) congestion externality (caused by both ride-sourcing and regular vehicles) is not considered (Yang et al., 2005).

\subsection{Matching Function}

The matchings between customers and drivers are completed via a matching algorithm implemented at the ride-sourcing platform. Taking Uber as an example, it dispatches one of the vehicles within a coverage radius of a requesting customer. The dispatching is made to minimize the estimated waiting time for the customer (Ranney, 2015). At the aggregate level, we assume that a matching function (a production function) can be used to characterize such a process. Note that aggregate matching functions have been calibrated for traditional street-hailing (e.g., Yang et al., 2014) or radio-dispatch taxi market (e.g., Schroeter, 1983). Although being much more efficient with a larger matching area and more complete information of drivers and customers, the matching technology offered by a ride-sourcing platform is actually similar to the one adopted by radio-dispatch taxi companies. We thus assume that that aggregate matching functions may still be valid for representing the matching technology, which is validated by our agent-based simulation study.

More specifically, we consider a stationary state where variables such as the numbers of waiting customers $\left(N^{c}\right)$ and vacant ride-sourcing vehicles $\left(N^{v t}\right)$ are time invariant. The matching function then relates the rate of matchings (more precisely, meetings) per hour $\left(m^{c-t}\right)$ to $N^{v t}$ and $N^{c}$ at any instant (Yang and Yang, 2011). Note $N^{v t}=w^{t} T^{v t}$ where $w^{t}$ is the average "searching" time for a driver before meeting a customer and $T^{v t}$ is the arrival rate of vacant vehicles per hour. $N^{c}=w^{c} Q$, where $w^{c}$ is the average customer waiting time and $Q$ is the customer demand per hour. The matching function can be formally written as:

$$
m^{c-t}=M\left(N^{v t}, N^{c}\right)=M\left(w^{t} T^{v t}, w^{c} Q\right)
$$

where $\partial M / \partial N^{v t}>0, \partial M / \partial N^{c}>0$ are assumed so that the increase of either vacant vehicles or waiting customers will increase the meeting rate.

We define the elasticities of the matching function with respect to $N^{v t}$ and $N^{c}$ as $\alpha_{1}$ and $\alpha_{2}$, respectively. The elasticities reflect the matching technology of the ride-sourcing platform. We hereinafter assume them to be constant and within the range of $[0,1]$. This assumption leads to a Cobb-Douglas matching function:

$$
m^{c-t}=A\left(N^{v t}\right)^{\alpha_{1}}\left(N^{c}\right)^{\alpha_{2}}
$$

where $A$ is a scaling parameter, which depends on the unit of the meeting rate and encapsulates other factors in the matching technology that are not fully captured by $\alpha_{1}$ and $\alpha_{2}$. Moreover, the parameter can be interpreted to be related to the area of the ride-sourcing market and the cruising speed of vacant vehicles (we assume waiting customers remain stationary until being picked up). 
To see this, consider the following customers' average waiting time function, which is obtained by considering $m^{c-t}=Q=T^{v t}$ at the stationary state together with Eqs. (1)-(2):

$$
w^{c}=(Q)^{\frac{1-\alpha_{1}-\alpha_{2}}{\alpha_{2}}}(A)^{-\frac{1}{\alpha_{2}}}\left(w^{t}\right)^{-\frac{\alpha_{1}}{\alpha_{2}}}
$$

Setting $\alpha_{2}=1$ yields:

$$
w^{c}=\frac{1}{A\left(Q w^{t}\right)^{\alpha_{1}}}=\frac{1}{A\left(N^{v t}\right)^{\alpha_{1}}}
$$

The above implies that customers' waiting time only depends on the number of vacant vehicles. This is true particularly when the supply of vacant vehicles is more than sufficient to serve the customers and there is no competition among customers over a particular vehicle. Further assuming $\alpha_{1}=1$ and $\alpha_{1}=0.5$, Eq. (4) will be reduced to the waiting time functions derived by Douglas (1972) and Arnott (1996) for cruising and radio dispatching taxi market respectively where $A$ represents the area of the market divided by the running speed of vacant vehicles.

The matching function is increasing, constant or decreasing returns to scale when the sum of $\alpha_{1}$ and $\alpha_{2}$ is larger than, equal to or smaller than one (Yang and Yang, 2011). By analyzing the radio-dispatch taxi market in Minneapolis, U.S. and the overall taxi market in Hong Kong, China, respectively, both Schroeter (1983) and Yang et al. (2014) reported increasing-returns-toscale matching functions. The degree varies from 1.13 to 1.16 . The increasing-returns-to-scale property is commonly seen in a queueing process that exists in many transportation systems (Mohring, 1972; Schroeter, 1983; Arnott, 1996; Yang and Yang, 2011). In a ride-sourcing market, drivers often cruise to "hotspots" in order for being matched early. Higher densities of both customers and drivers increase the matching probability. After being matched, the matched driver will have to travel to pick up the customer, and the average travelling distance decreases with the increases in the numbers of customers and drivers. Due to the economy of density, the average waiting times of customers and drivers is expected to decrease more than linearly if both the numbers of customers and drivers increase. Consequently, the meeting rate increases more than linearly with the numbers of customers and drivers. This observation has also been confirmed in an agent-based simulation study we conducted. Therefore, the analyses hereinafter primarily focus on cases with an increasing-returns-to-scale matching function.

\subsection{Customer Demand}

Consider a stationary state where the hourly demand of customers (passengers) is $\bar{Q}$. Each customer consumes exactly one trip and faces two transportation modes: the ride-sourcing service and alternative modes such as transit. The customer derives a deterministic utility $V_{0}$ from completing the trip while incurring a generalized $\operatorname{cost} \mu$ for using the ride-sourcing service and $C$ for the other options. Note $\mu$ is determined endogenously while $C$ is exogenously given 
as a constant. With error terms $\varepsilon_{R}, \varepsilon_{O}$ capturing unmeasurable attributes, the utility from utilizing each mode can thus be specified as follows:

$$
\begin{aligned}
& U_{R}=V_{0}-\mu+\varepsilon_{R} \\
& U_{O}=V_{0}-C+\varepsilon_{O}
\end{aligned}
$$

where $\mu=F+\beta w^{c}+\tau l$, consisting of the trip fare $(F)$, waiting time cost $\left(\beta w^{c}\right)$ and in-vehicle travel time cost $(\tau l)$. This specification implicitly assumes that customers are homogenous in their values of waiting time $(\beta)$ and in-vehicle travel time $(\tau)$. Moreover, $w^{c}$ is determined endogenously in Eq. (3) while $l$ represents the average trip time and is assumed constant. Each customer is assumed to choose the option that maximizes his utility. Consequently, the demand for the ride-sourcing service $(Q)$ will depend on the distribution of the error terms but can be specified as a decreasing function of the generalized cost:

$$
Q=f(\mu)=f\left(F+\beta w^{c}+\tau l\right)
$$

where $f^{\prime}<0$ over the domain $\mu>0$.

\subsection{Comparative Statics}

At any instant of the stationary state, total vehicle equals the sum of the numbers of vacant vehicles $N^{v t}$ and occupied vehicles $N^{o}$, i.e., $N=N^{v t}+N^{o}=Q w^{t}+Q l$. The matching function

essentially dictates the form of the waiting time function $\overline{W^{c}}\left(Q, w^{t}\right)$, as defined in Eq. (3). So far, we have identified the following three equations:

$$
\begin{aligned}
& w^{c}=\overline{W^{c}}\left(Q, w^{t}\right) \\
& Q=f\left(F+\beta w^{c}+\tau l\right) \\
& N=Q\left(w^{t}+l\right)
\end{aligned}
$$

The unknowns are $Q, F, w^{t}, w^{c}$ and $N$. Treating $F$ and $N$ as decision variables, we present some derivatives with respect to $F$ and $N$ as follows:

$$
\begin{aligned}
& \frac{\partial Q}{\partial F}=\frac{f^{\prime}}{1-f^{\prime} \beta \overline{W_{1}^{c}}+f^{\prime} \beta \overline{W_{2}^{c}} \frac{N}{Q^{2}}} \\
& \frac{\partial Q}{\partial N}=\frac{f^{\prime} \beta \frac{1}{Q} \overline{W_{2}^{c}}}{1-f^{\prime} \beta \overline{W_{1}^{c}}+f^{\prime} \beta \overline{W_{2}^{c}} \frac{N}{Q^{2}}}
\end{aligned}
$$


where $\overline{W_{1}^{c}}=\frac{1-\alpha_{1}-\alpha_{2}}{\alpha_{2}} \frac{w^{c}}{Q}, \overline{W_{2}^{c}}=-\frac{\alpha_{1}}{\alpha_{2}} \frac{w^{c}}{w^{t}}$. It can be shown that

$$
\partial Q / \partial F<0, \quad \partial Q / \partial N>0
$$

Similarly, we have:

$$
\begin{aligned}
& \frac{\partial w^{t}}{\partial F}=-N \frac{1}{Q^{2}} \frac{\partial Q}{\partial F} \\
& \frac{\partial w^{c}}{\partial F}=\left(\overline{W_{1}^{c}}-\overline{W_{2}^{c}} \frac{N}{Q^{2}}\right) \frac{\partial Q}{\partial F} \\
& \frac{\partial w^{t}}{\partial N}=\frac{1}{Q^{2}}-\frac{N}{Q^{2}} \frac{\partial Q}{\partial N} \\
& \frac{\partial w^{c}}{\partial N}=\overline{W_{1}^{c}} \frac{\partial Q}{\partial N}+\overline{W_{2}^{c}}\left(\frac{1}{Q^{2}}-\frac{N}{Q^{2}} \frac{\partial Q}{\partial N}\right)
\end{aligned}
$$

where $\overline{W_{1}^{c}}, \overline{W_{2}^{c}}, \frac{\partial Q}{\partial F}, \frac{\partial Q}{\partial N}$ are defined in Eqs. (11) and (12).

\section{Market Scenarios of Single Ride-Sourcing Platform}

In this section, three market scenarios with a single ride-sourcing platform are examined. The monopoly scenario captures the platform's profit-maximizing behavior without any regulatory intervention. The first-best solution maximizes social welfare, but the platform and its drivers may be in deficit. We thus examine the second-best scenario by adding additional constraints to guarantee the reservation profits of the platform and drivers.

\subsection{Monopoly Scenario}

In this scenario, the ride-sourcing platform determines the trip fare and commission to attract both drivers and customers to the platform to facilitate their matchings in order to maximize the profit of the platform. Due to the static nature of our model, advanced pricing features such as surge pricing are not considered. The platform essentially provides a two-sided market and its decision making can be described as a leader-followers game where the monopoly platform serves as the leader who determines the trip fare $F$ and the commission $P$ to maximize its profit while customers and drivers are the followers. The former decides whether to use the ridesourcing service while the latter decide whether to provide the service. With a free entry and sufficient labor supply, drivers will do so until their profit reaches zero. The platform's problem can thus be written as follows: 


$$
\begin{array}{r}
\text { (P1) } \max _{F \geq 0, P \geq 0, N \geq 0} \pi_{P}=P Q-C_{P}(Q) \\
\text { s.t. }(F-P) Q-c N=0
\end{array}
$$

where $C_{P}(Q)$ is the cost function of the ride-sourcing platform and $c$ captures the average hourly operation cost of a vehicle and the opportunity cost of the driver. For simplicity, we hereinafter do not consider non-negative constraints of decision variables and only focus on interior solutions. Define the Lagrangian function of P1 as follows:

$$
L=\pi_{P}-\lambda[(F-P) Q-c N]
$$

The first-order necessary conditions (FONCs) of P1 yield:

$$
\begin{aligned}
& \lambda=-1 \\
& F=C_{P}{ }^{\prime}(Q)+c\left(w^{t}+l\right)+\frac{1-\alpha_{1}-\alpha_{2}}{\alpha_{1}} c w^{t}-\frac{Q}{f^{\prime}} \\
& c=\beta \frac{\alpha_{1}}{\alpha_{2}} \frac{w^{c}}{w^{t}}
\end{aligned}
$$

Eq.(21) indicates the profit of the drivers and that of the platform are substitutes while Eq. (23) shows $w^{c}$ is proportional to $w^{t}$ at optimality. Eq. (22) is the monopoly pricing formula. Define the price elasticity of demand as $\eta=-f^{\prime} \frac{F}{Q}>0$. It can be revised as:

$$
F-C_{P}^{\prime}(Q)-c\left(w^{t}+l\right)-\frac{1-\alpha_{1}-\alpha_{2}}{\alpha_{1}} c w^{t}=\frac{F}{\eta}
$$

which follows the form of the Lerner formula (Lerner, 1934). The right-hand-side of the pricing formula in Eq. (22) consists of four terms: the marginal cost of the platform $\left(C_{P}{ }^{\prime}(Q)\right)$, the average cost for a driver to serve a new customer $\left(c\left(w^{t}+l\right)\right)$, a "matching externality" ( $\left.\frac{1-\alpha_{1}-\alpha_{2}}{\alpha_{1}} c w^{t}\right)$ and the monopoly mark-up $\left(-\frac{Q}{f^{\prime}}\right)$. The "matching externality" is closely related to the returns to scale of the matching function. As will be shown shortly, the realized waiting (or searching) frictions are lower if the matching function is increasing returns to scale. The incoming customer will thus be charged lower compared to the cases when the matching function is constant or decreasing returns to scale. Such an externality also exists in the optimal pricing formulae in other investigated scenarios.

When substituting $P$ in the objective function using Eq. (19), we have the following equivalent formulation to $\mathrm{P} 1$ :

$$
\text { (P2) } \max _{N \geq 0, F \geq 0} \pi=F Q-c N-C_{P}(Q)
$$




$$
\text { s.t. } F-\frac{c N}{Q} \geq 0
$$

which indicates the platform maximizes its joint profit with its drivers. In fact, Eq. (26) can be safely dropped if we assume the resulting profit $\pi$ is nonnegative at optimality. (See Appendix A for more information). This implies that, albeit not owning any vehicle, the platform essentially behaves like a traditional cab company that has a monopoly on the ride-for-hire market and thus determines its price and fleet size to maximize its profit.

\subsection{First-Best Scenario}

The first-best scenario represents an ideal case where a social planner or the platform maximizes total social welfare instead of its own profit by deciding the trip fare and fleet size. The commission does not impact social welfare but the revenue sharing between drivers and the platform. Therefore, the welfare formulation is similar to that in the traditional taxi literature except the additional cost incurred by the platform (Yang and Yang, 2011). The corresponding maximization problem can be written as:

$$
\text { (P3) } \max _{F \geq 0, N \geq 0} S=\int_{0}^{Q} f^{-1}(z) d z-Q\left(\beta w^{c}+\tau l\right)-c N-C_{P}(Q)
$$

The FONC of the above problem leads to:

$$
\begin{aligned}
& F-C_{P}^{\prime}(Q)-c\left(w^{t}+l\right)-\frac{1-\alpha_{1}-\alpha_{2}}{\alpha_{1}} c w^{t}=0 \\
& c=\beta \frac{\alpha_{1}}{\alpha_{2}} \frac{w^{c}}{w^{t}}
\end{aligned}
$$

In a regular taxi market where the taxi trip production is increasing returns to scale, the firstbest pricing only covers the cost when a taxi is occupied and thus drivers' profit is negative (Douglas, 1972; Arnott, 1996; Cairns and Liston-Heyes, 1996). Utilizing Eq. (28), the joint hourly profit obtained by the ride-sourcing platform and its drivers is given as:

$$
\pi^{f-b}=F Q-c N-C_{P}(Q)=\left(\frac{1-\alpha_{1}-\alpha_{2}}{\alpha_{1}} c N^{v t}\right)+\left(C_{P}^{\prime}(Q) Q-C_{P}(Q)\right)
$$

Define the elasticity of the cost function as $\eta_{c}=C_{P}{ }^{\prime}(Q) \frac{Q}{C_{P}(Q)}$. If $\alpha_{1}+\alpha_{2}>1$ and $\eta_{c} \leq 1$, then $\pi^{f-b}<0$. That is, if the matching function exhibits increasing returns to scale and the cost function of the platform shows economies of scale, the profits for the platform and drivers will be negative, making the first-best solution unsustainable. 


\subsection{Second-Best Scenario}

Since the profits for the platform and its drivers may be in deficit in the first-best scenario, we consider the following second-best pricing.

$$
\text { (P4) } \begin{array}{rl}
\max _{F \geq 0, N \geq 0, P \geq 0} & S=\int_{0}^{Q} f^{-1}(z) d z-Q\left(\beta w^{c}+\tau l\right)-c N-C_{P}(Q) \\
\text { s.t. } & P Q-C_{P}(Q) \geq \pi_{P}^{o} \\
& (F-P) Q-c N \geq 0
\end{array}
$$

where $\pi_{P}^{o}$ represents the reservation profit of the platform and is nonnegative. Define $\pi_{P}=P Q-C_{P}(Q), \pi_{D}=(F-P) Q-c N$, the Lagrangian function associated with $\mathrm{P} 4$ can be written as :

$$
L=S+\lambda_{P}\left(\pi_{P}-\pi_{P}^{o}\right)+\lambda_{D} \pi_{D}
$$

The FONC will then give:

$$
\begin{aligned}
& \lambda_{P}=\lambda_{D}(=\lambda) \\
& c=\beta \frac{\alpha_{1}}{\alpha_{2}} \frac{w^{c}}{w^{t}} \\
& F-C_{P}{ }^{\prime}(\mathrm{Q})-c\left(w^{t}+l\right)-\frac{1-\alpha_{1}-\alpha_{2}}{\alpha_{1}} c w^{t}=-\frac{\lambda}{1+\lambda} \frac{Q}{f^{\prime}}(\lambda \geq 0)
\end{aligned}
$$

As shown in Eq. (35), the Lagrangian multipliers associated with the reservation profit constraints are the same. We can thus sum up Eqs. (32) and (33) to bound the joint profit of the platform and its drivers. Eq. (37) follows the Ramsey pricing (Oum and Tretheway, 1988; Yang et al., 2005). It can be seen as a convex combination of the pricing formulas in the first-best and monopoly solutions. Further, the reservation profit constraints are binding given that the matching function exhibits increasing returns to scale. We summarize the results in the following proposition.

Proposition 1. If $\alpha_{1}+\alpha_{2}>1$ and $\eta_{c} \leq 1$, then $\pi_{P}=\pi_{P}^{o}, \pi_{D}=0$.

The proof of Proposition 1 is given in Appendix B. In our modeling framework, the commission $P$ serves as an instrument to split the revenue obtained by the platform and its drivers. Given a solution of $F$ and $N$ (and thus $Q$ ), $P$ is appropriately defined by the reservation profit constraints.

When the matching function shows increasing returns to scale, $\mathrm{P} 4$ is equivalent to the program that maximizes customers' demand subject to the same constraints (Douglas, 1972; Vany, 1975; Frankena, 1983; Frankena and Pautler, 1986; Yang et al., 2002). 


\subsection{Discussions on the Single Platform}

\subsubsection{Effects of Homogenous Value of Time}

We have investigated the optimality conditions of three different scenarios. Despite the difference in the pricing formula, the optimality conditions all unveil the fact:

$$
c=\beta \frac{\alpha_{1}}{\alpha_{2}} \frac{w^{c}}{w^{t}}
$$

which means the average customer waiting time is proportional to the average driver searching time at optimality. Figure 1 illustrates an economic intuition, following Yang and Yang (2011). The meetings between customers and drivers can be viewed as the production of the ridesourcing platform with inputs $N^{c}$ and $N^{v t}$. The monopolist and the social planner essentially differ in the chosen meeting rates (realized demand levels). At each demand level, the optimal matching is characterized by cost-minimizing production of the ride-sourcing platform. Therefore, the proportional relationship in Eq. (38) results from the tangency of the matching function and the total external cost curve. The assumption on the homogenous value of time of the customers guarantees the external cost curve is a line, presenting a constant slope for all the scenarios examined ${ }^{1}$. When the heterogeneity of value of time is modelled, however, such a tangency condition generally does not hold (See Appendix C for a detailed discussion).

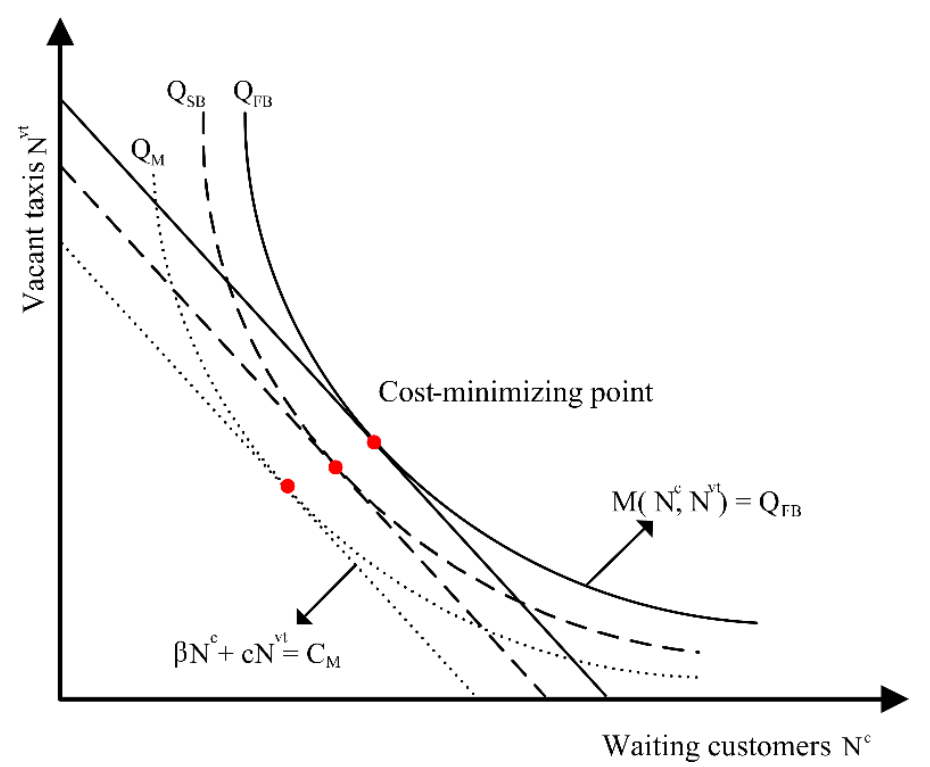

Figure 1 Cost Minimization of the Matching Technology.

\footnotetext{
${ }^{1}$ The effect of homogenous value of time is most evident when the matching function exhibits constant returns to scale. With the tangency condition, it is straightforward to show the average waiting and searching times are constant (Yang and Yang, 2011). The average waiting and searching times often serve as the "quality" measurement. Consequently, the monopolist and social planner will provide the same "service quality" if the customers are homogenous in their value of time (Spence, 1975; Yang and Yang, 2011).
} 


\subsubsection{Contract Solution Set and Properties}

The solution set for all the investigated cases are conceptually displayed in Figure 2 . The monopoly and first-best solutions are marked as the end points $M$ and $S$, respectively along a contract curve. The remaining points on the curve correspond to the second-best solution of varying joint profit levels between the platform and its drivers. Each point on the contract curve is characterized by the triple tangency of the joint profit contour, consumers' surplus contour and social welfare contour (Spence, 1975). That is, for a given reservation profit, the associated point maximizes the total welfare and vice versa.

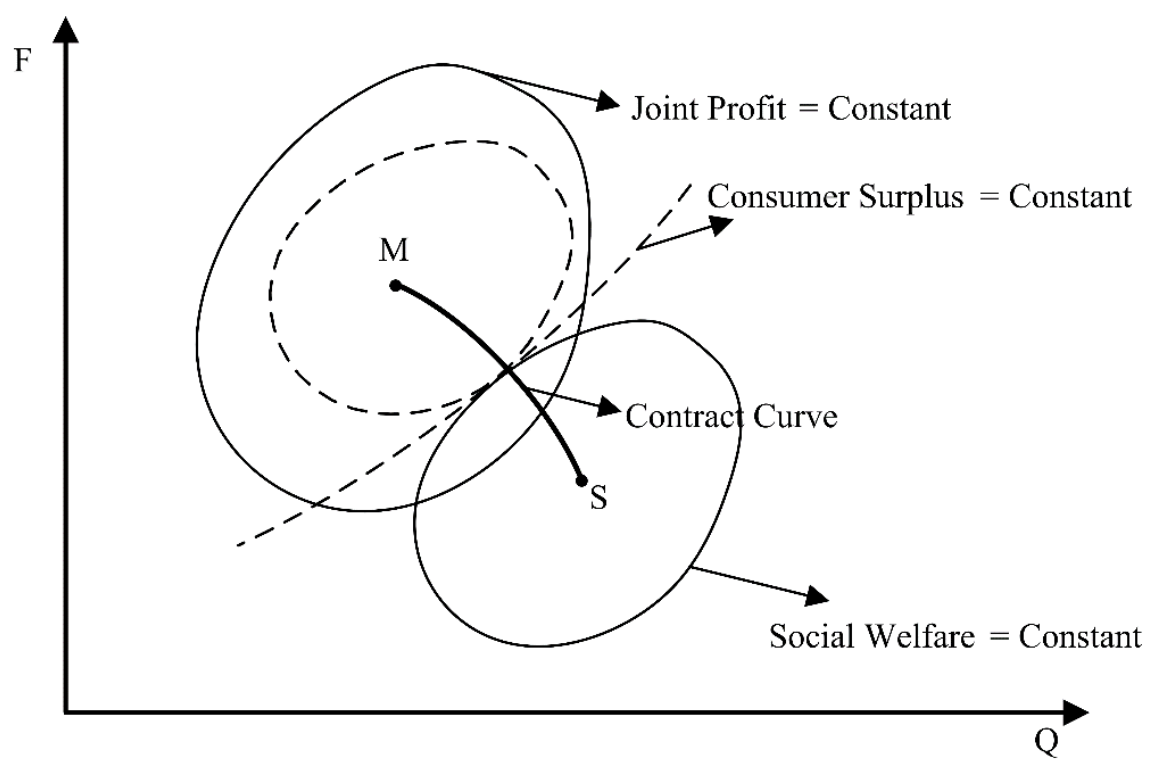

Figure 2 Contract Solution Set for Single Ride-Sourcing Platform.

Define the average joint profit as $\bar{\pi}=\frac{F Q-c N-C_{P}(Q)}{Q}$. It is intriguing to investigate the state where the increase of $Q$ will be mutually beneficial to all the participants, i.e., $\frac{d \bar{\pi}}{d Q}>0$ and $\frac{d w^{c}}{d Q}<0$ (Yang and Yang, 2011). Consider the process of gradually increasing $Q$ from the monopoly to the first-best solution, it is generally expected the total joint profit keeps decreasing (and so does the average joint profit, i.e. $\frac{d \bar{\pi}}{d Q}<0$ ) while the social welfare is strictly increasing ${ }^{2}$. In fact, the contract curve is characterized by Eqs. (8)-(10) together with Eq. (38). Total differentiating Eqs. (8) and (38) yields:

\footnotetext{
${ }^{2}$ Parallel to Proposition 1 in Yang and Yang (2011), we can treat $F, Q$ as decision variables and evaluate $\partial \bar{\pi} / \partial Q$ and $\partial w^{c} / \partial Q$. It can be shown the mutually beneficial situation occurs only when the matching function shows increasing returns to scale and the waiting time elasticity of demand is sufficiently large. However, such a solution is not on the contract curve and thus not of particular interest here.
} 


$$
\begin{aligned}
& d w^{c}=\overline{W_{1}^{c}} d Q+\overline{W_{2}^{c}} d w^{t}=\frac{1-\alpha_{1}-\alpha_{2}}{\alpha_{2}} \frac{w^{c}}{Q} d Q-\frac{\alpha_{1}}{\alpha_{2}} \frac{w^{c}}{w^{t}} d w^{t} \\
& \beta \alpha_{1} d w^{c}=c \alpha_{2} d w^{t}
\end{aligned}
$$

Eliminating $d w^{t}$ and re-arranging the terms, we have:

$$
\frac{d w^{c}}{d Q}=\frac{1-\alpha_{1}-\alpha_{2}}{\left(\alpha_{1}+\alpha_{2}\right)} \frac{w^{c}}{Q}
$$

which is negative given the matching function shows increasing returns to scale.

\subsection{Regulation Policies}

As previously shown, the monopoly ride-sourcing market is suboptimal in terms of social welfare and thus regulation may be necessary. This section seeks for regulation strategies by assuming the regulator has complete information. We examine possible combinations of regulatory variables to identify the most efficient regulation strategy that induces the second-best and requires the minimum number of regulatory variables. The potential regulation variables include $P, F, N$.

Let $F^{S B}, N^{S B}, P^{S B}$ solve P4. Regulating all three variables definitely works. Since the breakeven constraint for drivers is binding at the second-best solution as given in Proposition 1, together with Eqs. (8)-(10), the nonlinear equation system has only two degrees of freedom (four equations and six unknowns). Therefore, regulating any two of these three variables at the second-best level should work.

Below we show that properly regulating the commission charged by the platform can also yield the second best. To see this, recall that the second-best solution can also be achieved by maximizing the customer demand subject to the reservation profit constraint. If the commission is regulated, the proprietary ride-sourcing platform will maximize the customer demand, given that the cost function of the platform is assumed to exhibit economies of scale. Therefore, a proper choice of commission $P$ by the regulator would trigger the platform to maximize its profit to the reservation level, leading the final solution to coincide with the second-best one. Mathematically, when only $P$ is regulated at the second-best level, the FONC of P1 is given by $c=\beta \frac{\alpha_{1}}{\alpha_{2}} \frac{w^{c}}{w^{t}}$ and $\left(F-P^{S B}\right) Q-c N=0$. Correspondingly we have $c=\beta \frac{\alpha_{1}}{\alpha_{2}} \frac{w^{c}}{w^{t}}$ and $\left(F-P^{S B}\right) Q-c N=0$ from the FONC of P4. Both systems of equations have two unknowns $N, F$ and share the same equations. Therefore, they will have the same solution.

However, regulating $F$ or $N$ only will not work. 


\section{Regulating $F$}

When only restricting $F=F^{S B}$, the FONC of $\mathrm{P} 1$ is given by $F^{S B}=C_{P}{ }^{\prime}(Q)+c / \frac{\partial Q}{\partial N}$ and $\left(F^{S B}-P\right) Q-c N=0$. Correspondingly from the FONC of P4, we have $F^{S B}=C_{P}{ }^{\prime}(Q)+c / \frac{\partial Q}{\partial N}+\frac{1}{1+\lambda} \frac{Q}{f^{\prime}}$ and $\left(F^{S B}-P\right) Q-c N=0$. Both systems of equations have two unknowns: $N, P$, but they differ in one equation and thus generally admit different solutions.

\section{Regulating $N$}

When only restricting $N=N^{S B}$, the FONC of $\mathrm{P} 1$ is given by $F=C_{P}{ }^{\prime}(Q)-\frac{Q}{f^{\prime}}+\beta \overline{W_{1}^{c}} Q-\beta \overline{W_{2}^{c}} \frac{N^{S B}}{Q}$ and $(F-P) Q-c N^{S B}=0$. Correspondingly from the FONC of $\mathrm{P} 4$, we have $F=C_{P}{ }^{\prime}(Q)-\frac{\lambda}{1+\lambda} \frac{Q}{f^{\prime}}+\beta \overline{W_{1}^{c}} Q-\beta \overline{W_{2}^{c}} \frac{N^{S B}}{Q}$ and $(F-P) Q-c N^{S B}=0$. Following the same argument as above, we conclude that regulating $N$ only may not achieve the second best.

In summary, we have the following proposition.

Proposition 2. Assuming customers are homogenous in their value of time and the regulator has complete information, regulating the commission alone will achieve the second best if the matching function exhibits increasing returns to scale and the cost function of the ride-sourcing platform shows economies of scale.

Admittedly, the discussion so far has been based on the assumption of homogenous value of time for the customers. If a continuous distribution is adopted to capture the heterogeneity in the value of time, merely regulating the commission may not be enough. The main reason is that the tangency condition given in Eq. (38) may no longer hold. The regulator may then have to regulate two variables to achieve the second best.

\section{Competing Platforms}

The above analyses on a hypothetical single platform shed some light on the properties of the ride-sourcing market. However, the real-world situation is more complicated since several ridesourcing platforms are often competing for the market (e.g., Uber and Lyft in the U.S.). Some proponents of ride-sourcing companies have stated that competition may lower down the price level and improve social welfare. In this section, we investigate such a statement in a duopoly ride-sourcing market. We consider that a driver will only work for a particular platform and a customer only uses one platform for a particular trip. It is not uncommon that a customer or driver installs more than one ride-sourcing apps. Such a multi-homing issue is not considered in this paper (Rochet and Tirole, 2003; Armstrong, 2006).

With one more ride-sourcing platform, the utility function for a customer to choose each option is considered as: 


$$
\begin{aligned}
& U_{R_{i}}=V_{0}-\mu_{i}+\varepsilon_{R_{i}}, i \in 1,2 \\
& U_{O}=V_{0}-C+\varepsilon_{O}
\end{aligned}
$$

All the other specifications are the same as the single platform case. The demand functions of the two platforms depend on the distribution of the error terms but can generally be represented as $Q_{1}=f^{1}\left(\mu_{1}, \mu_{2}\right)$ and $Q_{2}=f^{2}\left(\mu_{1}, \mu_{2}\right)$. To facilitate the analysis, we make the following assumptions:

Assumption 1. The own-price effect is negative: $f_{1}^{1}=\frac{\partial Q_{1}}{\partial \mu_{1}}<0, f_{2}^{2}=\frac{\partial Q_{2}}{\partial \mu_{2}}<0$. The cross-price effect is positive and symmetric: $f_{1}^{2}=\frac{\partial Q_{2}}{\partial \mu_{1}}=\frac{\partial Q_{1}}{\partial \mu_{2}}=f_{2}^{1}>0$. Further, $-f_{1}^{1}>f_{2}^{1}$ and $-f_{2}^{2}>f_{1}^{2}$. The above assumption generally holds for the demand functions of two competing firms.

Similarly, we assume a Cobb-Douglas matching function for each ride-sourcing platform. To simplify our analysis, we further assume the same parameter set $\left\{A, \alpha_{1}, \alpha_{2}\right\}$ as that in the single platform for each platform. Given the interpretation of the parameters in Section 2.1, we essentially assume that both platforms have the same geographic coverage of users, running speed of vehicles, and matching technology as the single platform. To summarize, we have the following assumption:

Assumption 2. The matching function for each ride-sourcing platform follows the CobbDouglas type with the same parameters as those being used for the single platform. Namely, $Q_{1}=A\left(N^{v t_{1}}\right)^{\alpha_{1}}\left(N^{c_{1}}\right)^{\alpha_{2}}$ and $Q_{2}=A\left(N^{v t_{2}}\right)^{\alpha_{1}}\left(N^{c_{2}}\right)^{\alpha_{2}}$.

\subsection{Competition between Ride-Sourcing Platforms}

As seen previously, the commission $P_{i}, i=1,2$ can be substituted into the objective function using the reservation profit constraint. It is positive with the assumption on the non-negativity of the joint profit between the platform and its drivers in a mature ride-sourcing market. We further assume both platforms choose $\left(F_{i}, N_{i}\right), i=1,2$ simultaneously for profit maximization and focus on the Nash equilibrium (NE). Let's consider Platform 1:

$$
\text { (P5) } \max _{F_{1} \geq 0, N_{1} \geq 0} \pi_{1}=F_{1} Q_{1}-c N_{1}-C_{P_{1}}\left(Q_{1}\right)
$$

By setting $\frac{\partial \pi_{1}}{\partial F_{1}}=0$ and $\frac{\partial \pi_{1}}{\partial N_{1}}=0$, we have:

$$
\left[F_{1}-C_{P_{1}}{ }^{\prime}\left(Q_{1}\right)\right] \frac{\partial Q_{1}}{\partial F_{1}}=-Q_{1}
$$




$$
\left[F_{1}-C_{P_{1}}^{\prime}\left(Q_{1}\right)\right] \frac{\partial Q_{1}}{\partial N_{1}}=-c
$$

Note that $\frac{\partial Q_{1}}{\partial F_{1}}=\frac{\delta_{1}}{1-\delta_{1} \Delta_{1}}$ and $\frac{\partial Q_{1}}{\partial N_{1}}=\frac{\delta_{1} \beta \overline{W_{2}^{c_{1}}} / Q_{1}}{1-\delta_{1} \Delta_{1}}$, where $\delta_{1}=\frac{f_{1}^{1}-\Delta_{2} f_{1}^{1} f_{2}^{2}+\Delta_{2} f_{1}^{2} f_{2}^{1}}{1-\Delta_{2} f_{2}^{2}}$ $\Delta_{i}=\beta\left[\overline{W_{1}^{c_{i}}}-\overline{W_{2}^{c_{i}}} \frac{N_{i}}{\left(Q_{i}\right)^{2}}\right], i=1,2$. Next, dividing Eq. (45) by Eq. (46) and substituting $\frac{\partial Q_{1}}{\partial F_{1}}$ and $\frac{\partial Q_{1}}{\partial N_{1}}$, we obtain:

$$
c=\beta \frac{\alpha_{1}}{\alpha_{2}} \frac{w^{c_{1}}}{w^{t_{1}}}
$$

Similar to the single-platform scenario, the tangency condition still holds, i.e., the costminimizing behavior of each competing platform yields a customer's waiting time being proportional to a driver's searching time.

Given Eq. (47), one can verify $\Delta_{i}=\frac{1}{Q_{i}}\left[\frac{1-\alpha_{1}-\alpha_{2}}{\alpha_{1}} c w^{t_{i}}+c\left(w^{t_{i}}+l\right)\right]>0, i=1,2$. Define the price elasticity of $Q_{1}\left(\mu_{1}, \mu_{2}\right)$ as $\eta_{1}=-f_{1}^{1} \frac{F_{1}}{Q_{1}}>0$. Eq. (45) can be spelled out as:

$$
F_{1}-C_{P_{1}}^{\prime}\left(Q_{1}\right)-Q_{1} \Delta_{1}=\frac{F_{1}}{\eta_{1}}\left(\frac{f_{1}^{1}}{\delta_{1}}\right)
$$

It is expected that $\eta>1$ and $\eta_{1}>1$ for the platform(s) to charge positive trip fares. The pricing formula in Eq. (48) is of similar form to that in Eq. (24), apart from being multiplied by a scaling factor $f_{1}^{1} / \delta_{1}$. It is strictly larger than one given $f_{1}^{1}<0$ and $-\Delta_{2} f_{1}^{1} f_{2}^{2}>\Delta_{2} f_{1}^{2} f_{2}^{1}>0$. The ratio of price levels under duopoly and monopoly is given as follows:

$$
\frac{F_{1}}{F}=\frac{\bar{C}_{P}+\frac{1-\alpha_{2}}{\alpha_{1}} c w^{t_{1}}+c l}{\bar{C}_{P}+\frac{1-\alpha_{2}}{\alpha_{1}} c w^{t}+c l} \frac{\eta_{1} \eta-\eta_{1}}{\eta_{1} \eta-\eta\left(\frac{f_{1}^{1}}{\delta_{1}}\right)}
$$

where the platform production cost is assumed linear, i.e. $C_{P_{1}}{ }^{\prime}\left(Q_{1}\right)=C_{P}{ }^{\prime}(Q)=\bar{C}_{P}$. For a symmetric NE, it is expected $Q_{1} \leq Q$. Similar to Eqs. (39)-(41), the average waiting and searching times increase as the decrease of the platform specific demand given the matching function has increasing returns to scale. It follows that $w^{t_{1}}>w^{t}\left(w^{c_{1}}>w^{c}\right)$, indicating more 
matching frictions. Accordingly, the first component in the RHS of Eqn. (49) is no less than one while the second component is related to the price elasticities of demand. Its value is unclear without fully specifying the demand function. If $\frac{\eta_{1}}{\eta}<\left(\frac{f_{1}^{1}}{\delta_{1}}\right)$, the price level under NE is strictly larger than that under the monopoly. Otherwise, the ratio of $F_{1}$ over $F$ remains indeterminate. Generally, one needs to explore the change of the price elasticity of demand and that of the matching friction. The conventional wisdom that competition lowers the price level does not stand if the increase of the former is dominated by the latter.

\subsection{Second Best for Dual Ride-Sourcing Platforms}

This section directly investigates the second-best outcome with the dual platforms. The firstbest solution can be obtained by setting the Lagrangian multiplier associated with the reservation profit constraint to zero in the optimality conditions of the second-best problem.

Define $V\left(\mu_{1}, \mu_{2}\right)$ as the customers' surplus from completing a trip. With certain regularity conditions (Sheffi, 1985), the following properties hold by construction:

$$
\frac{\partial V}{\partial \mu_{1}}=-Q_{1}, \frac{\partial V}{\partial \mu_{2}}=-Q_{2}
$$

To simplify the derivation, we treat $\left(\mu_{i}, N_{i}\right), i=1,2$ as the decision variables.

Mathematically, $\mu_{i} \equiv F_{i}+\beta w^{c_{i}}+\tau l, i=1,2$. The maximization program is formally written as:

$$
\begin{aligned}
& \text { (P6) } \max _{\mu>0, \mathbf{N} \geq 0} S=V\left(\mu_{1}, \mu_{2}\right)+\sum_{i=1}^{2}\left[F_{i} Q_{i}-c N_{i}-C_{P_{i}}\left(Q_{i}\right)\right] \\
& \text { s.t. } F_{i} Q_{i}-c N_{i}-C_{P_{i}}\left(Q_{i}\right) \geq \pi_{P_{i}}^{o}, i=1,2
\end{aligned}
$$

Define its Lagrangian function as follows:

$$
L(\boldsymbol{\mu}, \mathbf{N})=V\left(\mu_{1}, \mu_{2}\right)+\sum_{i=1}^{2}\left(\lambda_{i}+1\right)\left[F_{i} Q_{i}-c N_{i}-C_{P_{i}}\left(Q_{i}\right)\right]-\sum_{i=1}^{2} \lambda_{i} \pi_{P_{i}}^{o}
$$

By setting $\frac{\partial L}{\partial N_{i}}=0, i=1,2$, it is immediate to show:

$$
c=\beta \frac{\alpha_{1}}{\alpha_{2}} \frac{w^{c_{i}}}{w^{t_{i}}}, i=1,2
$$

which again follows the tangency condition. Next, setting $\frac{\partial L}{\partial \mu_{i}}=0, i=1,2$ yields: 


$$
\begin{aligned}
& (\lambda+1)\left(\gamma_{1} f_{1}^{1}+\gamma_{2} f_{1}^{2}\right)=-\lambda Q_{1} \\
& (\lambda+1)\left(\gamma_{1} f_{2}^{1}+\gamma_{2} f_{2}^{2}\right)=-\lambda Q_{2}
\end{aligned}
$$

where $\gamma_{i}=F_{i}-C_{P_{i}}{ }^{\prime}\left(Q_{i}\right)-Q_{i} \Delta_{i}, i=1,2$. Substituting $\gamma_{2}$ in Eq. (55), we then focus on the pricing formula for platform 1:

$$
\gamma_{1}=F_{1}-C_{P_{1}}{ }^{\prime}\left(Q_{1}\right)-Q_{1} \Delta_{1}=-\frac{\lambda_{1}}{\lambda_{1}+1} \frac{Q_{1}}{f_{1}^{1}} \sigma_{1}
$$

where $\sigma_{1}=\frac{\left(Q_{2} f_{1}^{2}\right) /\left(Q_{1} f_{2}^{2}\right)-1}{\left(f_{1}^{2} f_{2}^{1}\right) /\left(f_{1}^{1} f_{2}^{2}\right)-1}$. For more insight, further assume a symmetric solution where $Q_{1}=Q_{2}$ and $f_{1}^{1}=f_{2}^{2}$. Then $\sigma_{1}$ is reduced to $\frac{f_{1}^{1}}{f_{1}^{1}+f_{1}^{2}}$ which is strictly larger than one given Assumption 1. Define the price elasticity of $Q_{1}\left(\mu_{1}, \mu_{2}\right)$ as $\eta_{1}=-f_{1}^{1} \frac{F_{1}}{Q_{1}}>0$, Eq. (57) can be rewritten as:

$$
F_{1}-C_{P_{1}}{ }^{\prime}\left(Q_{1}\right)-Q_{1} \Delta_{1}=\frac{\lambda_{1}}{\lambda_{1}+1} \frac{F_{1}}{\eta_{1}} \sigma_{1}
$$

The pricing formula for platform 2 can be obtained by symmetry. The formula structure is the same as that in the single platform case, except being adjusted by the factor $\sigma_{1}$, which has its roots in platform collusion (a case where the two platforms cooperatively maximize the total profit). The fact that it is greater than one is by the nature of the pricing for substitute goods ${ }^{3}$.

The first-best pricing formula for the dual platforms can be obtained by setting $\lambda_{i}=0, i=1,2$ . Similarly, the first-best solution is not sustainable if the matching function is assumed to exhibit increasing returns to scale and the cost function of the platform has economies of scale. The second-best pricing formula can be thought of the convex combination of the first-best and the collusion formulas.

The solution sets obtained in the above scenarios can also be displayed over a contract curve that connects the social optimal (Point S) and NE solution (Point N) (Point C represents the solution that two platforms collude to maximize the joint profit. It corresponds to the monopoly solution). The remaining points on the contract curve represent a continuum of the second-best solutions characterized by different regulated profit levels. Define the average joint profit for

\footnotetext{
${ }^{3}$ Assume a market without friction or externality. Define $Q_{1}=f^{1}\left(F_{1}, F_{2}\right), Q_{2}=f^{2}\left(F_{1}, F_{2}\right)$ to be demand functions for two competing firms. Further assume $f_{1}^{1}<0, f_{2}^{2}<0, f_{2}^{1}=f_{1}^{2}>0$; the total cost for production is linear: $c Q_{1}, c Q_{2}$. The collusion pricing formula under the symmetric solution is: $F_{1}-c=\frac{F_{1}}{\eta_{1}} \frac{f_{1}^{1}}{f_{1}^{1}+f_{1}^{2}}$.
} 
platform $i \bar{\pi}_{i}, i=1,2$, one can verify $\frac{d \bar{\pi}_{i}}{d Q_{i}}<0, i=1,2$ for the solutions on the contract curve. $\frac{d w^{c_{i}}}{d Q_{i}}, i=1,2$ is strictly negative when the matching function shows increasing returns to scale.

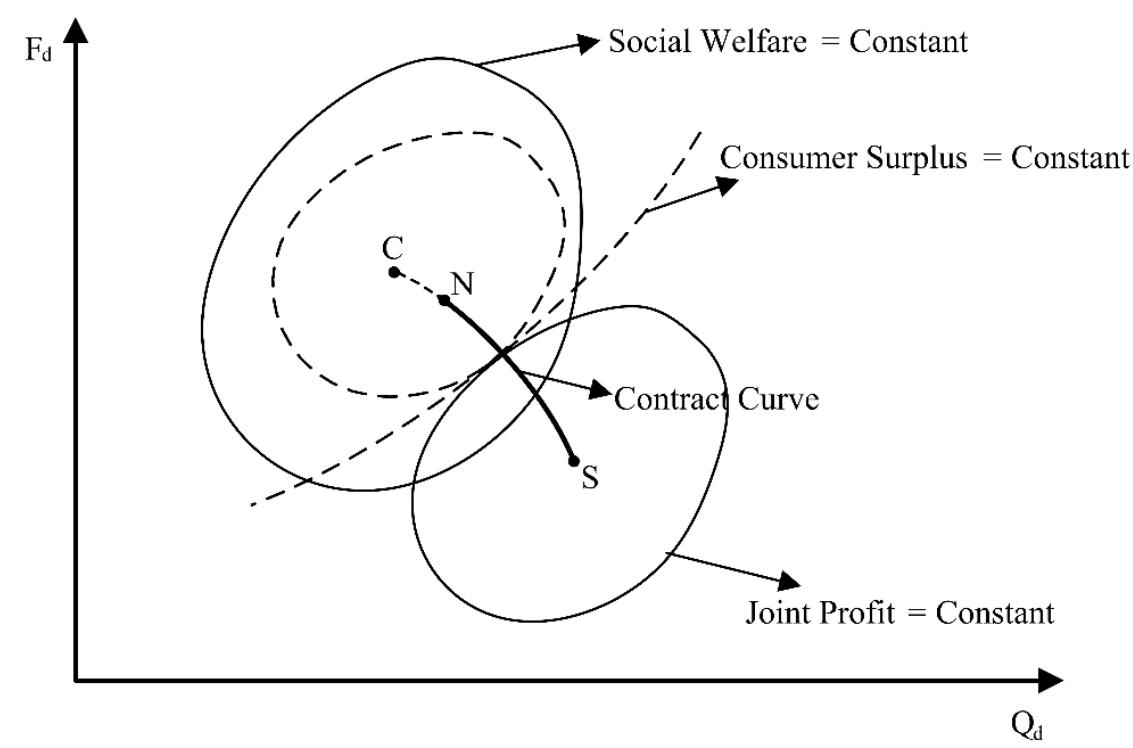

Figure 3 Contract Solution Set for Dual Ride-Sourcing Platforms.

\subsection{Welfare Changes between Single and Dual Platforms}

In this section, we attempt to investigate whether or not competition yields a more efficient market outcome and whether a regulated duopoly market is more efficient than a regulated monopoly market. To address the former, we compare the social welfare under the monopoly solution and duopoly solution. For the latter, we focus on the welfare change under the regulated second-best solutions.

To proceed, we specify the demand function by assuming that the error terms of the utility functions are identically and independently Gumbel distributed for both the single (Eqs. (5)-(6)) and dual platforms ${ }^{4}$ (Eqs. (42)-(43)). Therefore, the demand functions for the single platform and the other transportation modes are:

$$
Q=\frac{e^{-\theta \mu}}{e^{-\theta \mu}+e^{-\theta C}} \bar{Q}, Q=\frac{e^{-\theta C}}{e^{-\theta \mu}+e^{-\theta C}} \bar{Q}
$$

where $\theta>0$. For consistency, we assume the total base demand remains the same for both scenarios and the demand for the dual platforms are given as:

\footnotetext{
${ }^{4}$ The error terms are likely dependent in the duopoly market. This assumption is made for the ease of welfare comparison.
} 


$$
Q_{1}=\frac{e^{-\theta \mu_{1}}}{\Lambda} \bar{Q}, Q_{2}=\frac{e^{-\theta \mu_{2}}}{\Lambda} \bar{Q}, Q_{d}=\frac{e^{-\theta C}}{\Lambda} \bar{Q}
$$

where $\Lambda=e^{-\theta \mu_{1}}+e^{-\theta \mu_{2}}+e^{-\theta C}$.

In addition to specifying an increasing-return-to-scale matching function as highlighted in Assumption 2, we limit ourselves to the following aspects to further facilitate the comparison:

- The solutions for the dual ride-sourcing platforms are symmetric. The related variables are marked by the subscript $d$. e.g. $Q_{d}=Q_{1}=Q_{2}, w^{t_{d}}=w^{t_{1}}=w^{t_{2}}$.

- Total cost incurred by the platform is linear: $\bar{C}_{P} Q=C_{P}(Q)$.

\subsubsection{Analytical Comparison}

Utilizing the assumption on symmetric solution and linear platform cost, the welfare change between the single and dual platforms for a given market scenario is as follows:

$$
\begin{aligned}
\Delta S= & S_{d}-S_{s}=\left[\frac{1}{\theta} \ln \left(2 e^{-\theta \mu_{d}}+e^{-\theta C}\right)-\frac{1}{\theta} \ln \left(e^{-\theta \mu}+e^{-\theta C}\right)\right] \bar{Q}+ \\
& 2 Q_{d}\left[F_{d}-c\left(w^{t_{d}}+l\right)-\bar{C}_{P}\right]-Q\left[F-c\left(w^{t}+l\right)-\bar{C}_{P}\right]
\end{aligned}
$$

The first two terms measure the change in the consumers' surplus (Train, 2009) while the last two terms represent the change in producers' surplus, i.e., the joint profit between the platform and its drivers. For the duopoly and monopoly solutions, it is anticipated $2 Q_{d} \geq Q \geq Q_{d}$. For welfare evaluation, one needs to trade off the potential increase of consumers' surplus and the decrease from the joint profit.

Consider the extreme case that $Q_{d}$ approaches to $\frac{Q}{2}$ from the right. Then the change of consumers' surplus is approximately zero. Eq. (61) is reduced to:

$$
\begin{aligned}
\Delta S & =2 Q_{d}\left[F_{d}-F-c\left(w^{t_{d}}-w^{t}\right)\right] \\
& =2 Q_{d}\left[\ln 2-\left(\frac{\alpha_{1}+\alpha_{2}}{\alpha_{1}}\right) c\left(w^{t_{d}}-w^{t}\right)\right]
\end{aligned}
$$

One can verify $\Delta S \geq 0$ when $w^{t_{d}}-w^{t} \leq \frac{\alpha_{1}}{c\left(\alpha_{1}+\alpha_{2}\right)} \ln 2$. That is, competition is welfare improving if the increased searching friction is bounded above by a constant. The same condition holds when evaluating the welfare change at the second-best solutions (e.g. at zero profit level), where the change of the social welfare is equal to that of the consumers' surplus. Unfortunately, the explicit relationship between $w^{t_{d}}$ and $w^{t}$ (or equally $w^{c_{d}}$ and $w^{c}$ ) appears hard to obtain and neither has a closed form. We therefore resort to a numerical experiment to quantify the welfare changes. 


\subsubsection{Numerical Analysis}

Define $\Omega=\left\{\alpha_{1}, \alpha_{2}, A, C, \bar{Q}\right\}$ to be the experimental parameter set. $\alpha_{1}, \alpha_{2}, A$ are directly included in the matching function while $\bar{Q}$ and $C$ influence the base demand and realized demand splits. Since our focus is on the matching technology, the other parameters (e.g., $c, \beta)$ are held constant. In fact, it can be shown $\frac{\partial w^{t}}{\partial \alpha_{1}}<0, \frac{\partial w^{t}}{\partial \alpha_{2}}<0, \frac{\partial w^{t}}{\partial A}<0, \frac{\partial w^{t}}{\partial \bar{Q}}<0, \frac{\partial w^{t}}{\partial C}<0$ for all the solutions on the contract curve (The derivation for the sign of $\frac{\partial w^{t}}{\partial A}$ is given in Appendix D for illustration). Marginal increase of each of these parameters reduces the matching friction and thus indicates more efficient matching technology.

It is assumed $V_{0}=50(\$ /$ trip$), \beta=20(\$ / \mathrm{hr}), c=20(\$ / \mathrm{hr}), \theta=1(1 / \$), \tau=6(\$ / \mathrm{hr}), l=0.3(\mathrm{hr})$, $\bar{C}_{P}=2$ (\$/trip). For demonstration, we investigate two base demand levels: $\bar{Q}=100,000$ and 50,000(trip/hr) and the outside transportation costs: $C=19$ and 15 (\$/trip). For each combination of $\bar{Q}$ and $C$, a continuum of solutions are examined over a range of $\left(\alpha_{1}, \alpha_{2}, A\right)$. We explored three levels of returns to scale with $\alpha_{1}+\alpha_{2}=1.6,1.4,1.2$, respectively. For symmetry, $\alpha_{1}, \alpha_{2}$ are set equal.

The welfare changes between duopoly and monopoly solutions are given in Figure 5. For a meaningful comparison, we assume the joint platform demand will account for $10 \%-90 \%$ of the total passenger demand in the equilibrium states. This in turn indicates that the difference between the generalized cost of using the ride-sourcing service and the outside options cannot be very large. Accordingly, two dot curves are introduced to bound the solution region. Each solid red curve represents the computed welfare change over a feasible range of $A$ for a given elasticity pair $\left(\alpha_{1}, \alpha_{2}\right)$. The little bar at the left end of the curve means the location where no solution is available for either problem P1 or P5 if we further reduce $A$. The welfare difference curve slopes upwards, indicating $\Delta S$ increases as the increase of $A$. For a given $A$, the increase of elasticities $\left(\alpha_{1}, \alpha_{2}\right)$ generally yields larger $\Delta S$. 


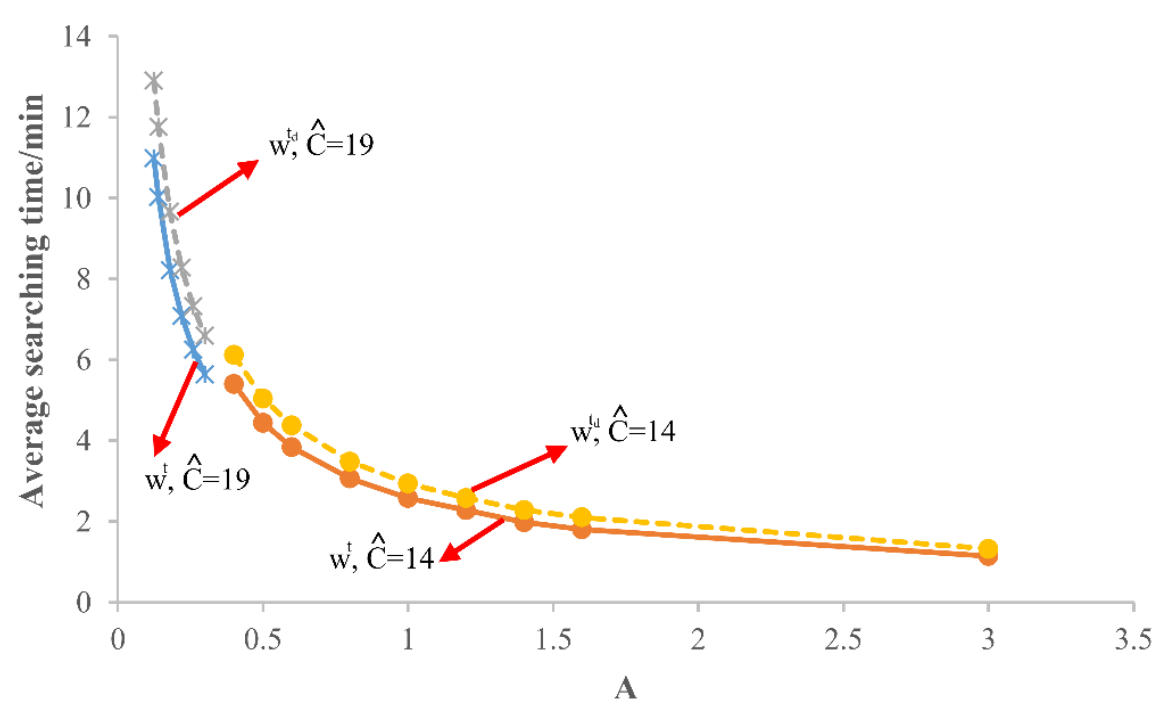

Figure 4 Average Searching Time $\left(\alpha_{1}=\alpha_{2}=0.7\right.$ and $\left.\bar{Q}=100000\right)$ 


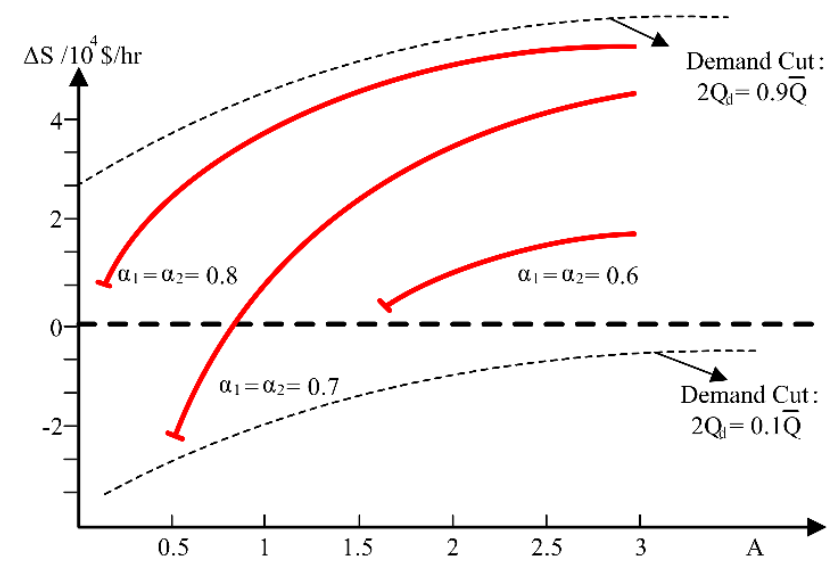

(a) $C=14, \bar{Q}=50000$ (unit: $\$ /$ trip, trip/hr)

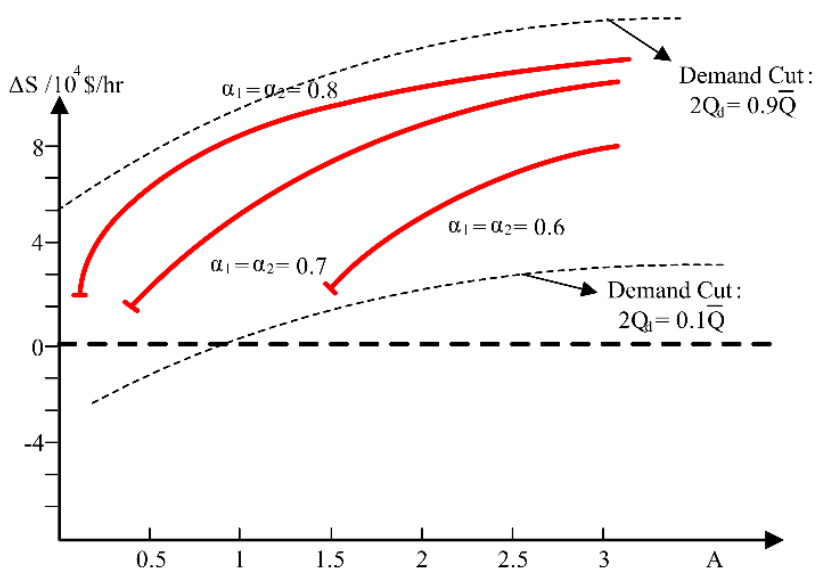

(b) $C=14, \bar{Q}=100000$ (unit: $\$ /$ trip, trip/hr)

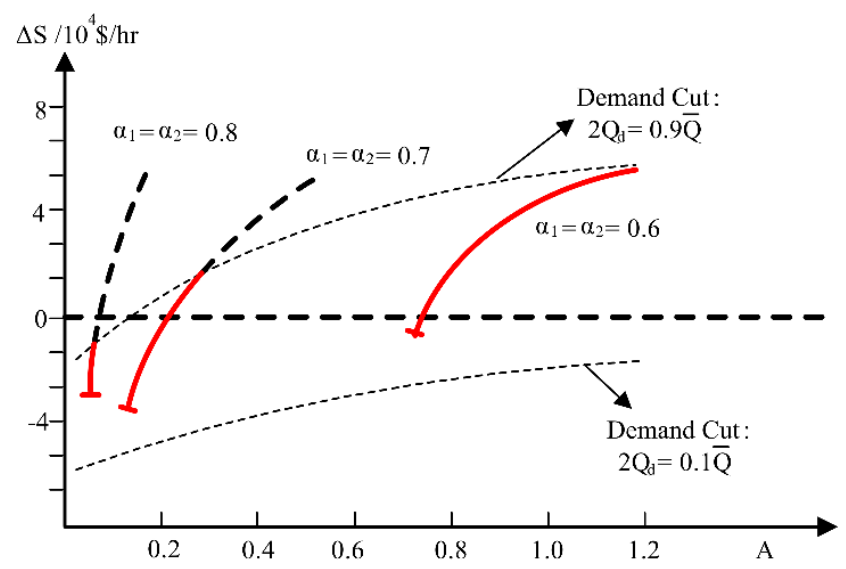

(c) $C=19, \bar{Q}=100000$ (unit: $\$ /$ trip, trip/hr)

Figure 5 Change in Social Welfare between Duopoly and Monopoly Solutions. 


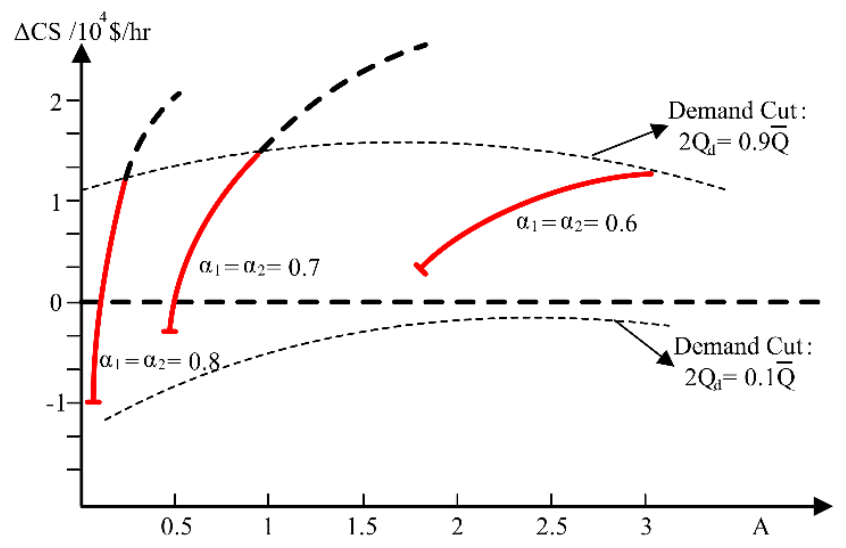

(a) $C=14, \bar{Q}=50000$ (unit: $\$ /$ trip, trip/hr)

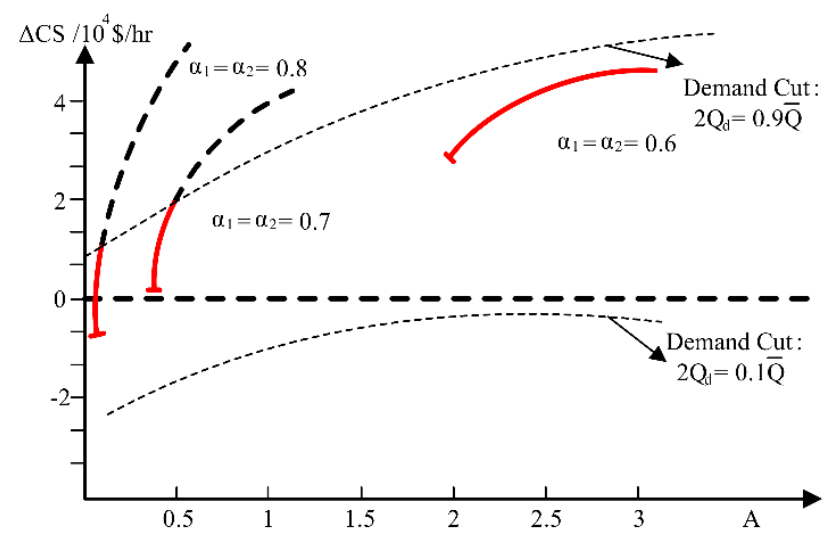

(b) $C=14, \bar{Q}=100000$ (unit: $\$ /$ trip, trip/hr)

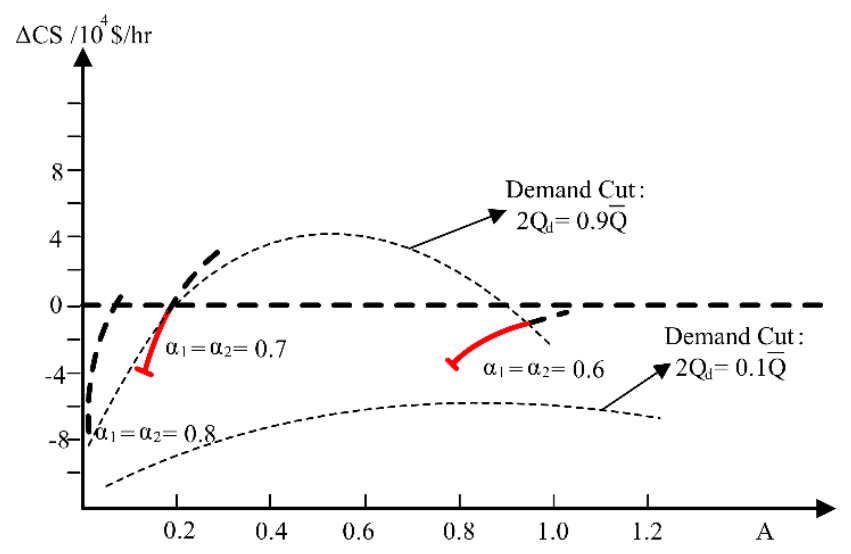

(c) $C=19, \bar{Q}=100000$ (unit: $\$ /$ trip, trip/hr)

Figure 6 Change of Consumers' Surplus between Dual and Single Platforms at Second-Best Solutions (at Zero Profit Level). 
In Figure 5(a), the changes of the total social welfare are positive but for the left end in the case of $\alpha_{1}=\alpha_{2}=0.7$. When the base demand increases from 50,000 to 100,000 (trip/hr), the total pattern moves upwards as seen in Figure 5(b). All the stable solutions in the region are characterized by $\Delta S>0$, indicating competition between the platforms is welfare improving. However, when $C$ increases from 14 to 19 (\$/trip) as shown in Figure 5(c), some left portion of all curves fall in the solution region where $\Delta S<0$. It should be pointed out that the welfare difference curve is plotted over the interval $(0.12,0.3)$ of $A$, which is different from Figure 5(a) and (b). In the cases shown in Figure 5(c), there are more matching frictions. Figure 4 shows the average searching times under duopoly and monopoly solutions when $\alpha_{1}=\alpha_{2}=0.7$ and $\bar{Q}=100,000$ (trip/hr). It can be observed that the average searching times for drivers are larger in duopoly than those in monopoly due to the decrease of platform-specific demands. For the cases presented in Figure 5(c) where competition is not efficient, the average search time is much higher, approximately 6-12 minutes.

The welfare comparison between the regulated dual and single platforms is given in Figure 6. The general tendency is similar to that in Figure 5. In many cases, competition increases social welfare. However in Figure 6(c), all the meaningful solutions are characterized by $\Delta S<0$. In those situations, a regulated monopoly platform will be superior to the regulated duopoly platforms in term of efficiency.

Although the numerical example is made from the arbitrary specification of function forms and parameter values, its interpretation is straightforward. Competition may not improve the social welfare when the matching technology is less efficient. Given the assumption of the increasing returns to scale of the matching function, its efficiency is not all about the advanced algorithms used by the ride-sourcing platform but also the size of the market, i.e., the number of users. To derive effective policies, the regulatory agency may need to obtain a good estimate on customers' demand function as well as the matching function. If the matching friction is large, the regulatory agency may rather encourage the merger of the ride-sourcing companies and then regulate them as a monopolist.

\section{Conclusion}

In this paper, we analyzed the ride-sourcing service using an aggregate model with the matchings between customers and drivers captured by an exogenous Cobb-Douglas matching function. We examined different market scenarios, solution properties and general economic outcomes of a hypothetical monopoly ride-sourcing market. It was found that without regulatory intervention the monopoly ride-sourcing platform would maximize the joint profits with its drivers. The first-best solution is not sustainable when the matching function is increasing returns to scale and the cost incurred by the platform exhibits economies of scale. Therefore, we further analyzed the second-best scenario with varying reservation profit levels for the ride-sourcing platform. In terms of market frictions, all the examined scenarios are characterized by a proportional relationship between the average waiting and searching times that implies the cost minimization of the matching production. In view of the market distortion, we demonstrated the feasibility of regulating two variables to achieve the second best. With the assumption of homogenous value of time of customers, we further showed regulating only the commission should guarantee the second best. 
To address the effects from platform competition, we extended our analysis by considering a duopoly setting. It was found that competition may not necessarily lower down the price levels when the increase of the matching friction overrides that of price elasticity of demand after the introduction of the competing platform. We further investigated the effects of competition on the welfare change when the matching function is increasing returns to scale. Based on the sensitivity analysis, we observed that competition can reduce social welfare when the matching technology is less efficient, and the increased matching friction for each platform dominates the surplus generated by having one additional option. In this case, the regulator may rather encourage the merger of the platforms and regulate them directly as a monopolist.

With the insights obtained from this paper, our future study will examine the impacts of ridesourcing platforms on the regular taxi industry by explicitly capturing the competition between the platforms and traditional cab companies. Rather than proposing regulations merely aimed at the ride-sourcing companies, we will consider a systematic reform of the regulation of the ridefor-hire market to make the competition more socially efficient. The investigation of this paper will also be advanced by considering heterogeneities of customers and drivers, and congestion externalities of ride-sourcing vehicles. Lastly, although the Cobb-Douglas matching function is flexible enough to capture the matching technology, its calibration requires empirical data. Moreover, the changes of its parameters are hard to predict when the function is applied to a new setting. We thus plan to adopt a deductive approach to delineate the matching process to derive the matching function.

\section{Acknowledgements}

The authors wish to express their gratitude to the three anonymous reviewers, whose constructive comments have improved the exposition of the paper. The work described in this paper was jointly supported by research grants from the National Science Foundation (CMMI-1362631; CMMI-1562420) and the Hong Kong Grant Council (Project No. HKUST-16222916). 


\section{References}

Anderson, D., 2014. "Not just a taxi”? For-profit ridesharing, driver strategies, and VMT.

Transportation 41, 1099-1117.

Armstrong, M., 2006. Competition in two-sided markets. The RAND Journal of Economics 37, 668-691.

Arnott, R., 1996. Taxi travel should be subsidized. Journal of Urban Economics 40, 316-333.

Badger, E., 2014. Taxi medallions have been the best investment in america for years. Now Uber may be changing that., The Washington Post.

http://www.washingtonpost.com/blogs/wonkblog/wp/2014/06/20/taxi-medallions-have-been-thebest-investment-in-america-for-years-now-uber-may-be-changing-that/. Accessed Jan 4, 2015.

Cairns, R.D., Liston-Heyes, C., 1996. Competition and regulation in the taxi industry. Journal of Public Economics 59, 1-15.

CPUC, 2013. Decision adopting rules and regulations to profect public safety while allowing new entrants to the transportation industry. The Public Utilities Commission of The State of California. http://docs.cpuc.ca.gov/PublishedDocs/Published/G000/M077/K192/77192335.PDF. Accessed Jan 3, 2015.

CPUC., 2014. Proposed decision of commissioner prevey. The Public Utilities of Commission of The State of California. http://docs.cpuc.ca.gov/PublishedDocs/Published/G000/M098/K126/98126852.PDF. Accessed Jan 2, 2015.

Douglas, G.W., 1972. Price regulation and optimal service standards: The taxicab industry. Journal of Transport Economics and Policy 6, 116-127.

ECMT, 2007. De/regulation of the taxi industry. OECD Publishing.

Frankena, M.W., 1983. The efficiency of public transport objectives and subsidy formulas. Journal of Transport Economics and Policy 17, 67-76.

Frankena, M.W., Pautler, P.A., 1986. Taxicab regulation: An economic analysis. Research in Law and Economics 9, 129-165.

He, F., Shen, Z.-J.M., 2015. Modeling taxi services with smartphone-based e-hailing applications. Transportation Research Part C: Emerging Technologies 58, Part A, 93-106.

Koopman, C., Mitchell, M., Thierer, A., 2015. The sharing economy and consumer protection regulation: The case for policy change. Mercatus Center at George Mason University.

Lerner, A.P., 1934. The concept of monopoly and the measurement of monopoly power. The Review of Economic Studies 1, 157-175. 
Mohring, H., 1972. Optimization and scale economies in urban bus transportation. The American Economic Review 62, 591-604.

Oum, T.H., Tretheway, M.W., 1988. Ramsey pricing in the presence of externality costs. Journal of Transport Economics and Policy, 307-317.

Pew Research Center, 2015. U.S. Smartphone use in 2015. http://www.pewinternet.org/2015/04/01/us-smartphone-use-in-2015/. Accessed Sep 2, 2015.

Ranney, M., 2015. Scaling Uber's real-time market platform. QCON London. http://www.slideshare.net/InfoQ/scaling-ubers-realtime-market-platform-50758747. Accessed Nov., 23, 2015.

Rayle, L., Shaheen, S., Chan, N., Dai, D., Cervero, R., 2014. App-based, on-demand ride services: Comparing taxi and ridesourcing trips and user characteristics in San Francisco University of California Transportation Center. UCTC-FR-2014-08.

Rochet, J.-C., Tirole, J., 2003. Platform competition in two-sided markets. Journal of the European Economic Association 1, 990-1029.

Schroeter, J.R., 1983. A model of taxi service under fare structure and fleet size regulation. The Bell Journal of Economics 14, 81-96.

Shaheen, S., 2014. Transportation network companies and ridesourcing. California Public Utilities Commission (CPUC) En Banc. http://www.cpuc.ca.gov/NR/rdonlyres/5C961222-B9C84E53-A54D-FC2A89C0A30C/0/RidesourcingCPUCShaheen_Final_v2.pdf. Accessed Jan 3, 2015.

Sheffi, Y., 1985. Urban Transportation Network: Equilibrium Analysis with Mathematical Programming Methods. Prentice Hall.

Spence, A.M., 1975. Monopoly, quality, and regulation. The Bell Journal of Economics 6, 417429.

Train, K.E., 2009. Discrete Choice Methods with Simulation. Cambridge University Press.

Vany, A.S.D., 1975. Capacity utilization under alternative regulatory restraints: An analysis of taxi markets. Journal of Political Economy 83, 83-94.

Yang, H., Wong, S.C., Wong, K.I., 2002. Demand-supply equilibrium of taxi services in a network under competition and regulation. Transportation Research Part B: Methodological 36, 799-819.

Yang, H., Yang, T., 2011. Equilibrium properties of taxi markets with search frictions. Transportation Research Part B: Methodological 45, 696-713.

Yang, H., Ye, M., Tang, W.H., Wong, S.C., 2005. Regulating taxi services in the presence of congestion externality. Transportation Research Part A: Policy and Practice 39, 17-40. 
Yang, T., Yang, H., Wong, S.C., Sze, N.N., 2014. Returns to scale in the production of taxi services: An empirical analysis. Transportmetrica A: Transport Science 10, 775-790. 


\section{Appendix}

\section{A. Relaxation of P2}

Let $F, N, P$ solves $\mathrm{P} 1$ and the optimal platform profit is $\pi_{P}(F, N, P)$. Considering the equivalence of $\mathrm{P} 1$ and $\mathrm{P} 2$, we know $F, N$ solves $\mathrm{P} 2$ with the optimal joint profit $\pi(F, N)$ . Note $\pi_{P}(F, N, P)=\pi(F, N)$ by construction. Let $F_{R}, N_{R}$ solves the relaxation of $\mathrm{P} 2$ in which Eq. (26) is dropped and the optimal joint profit is $\pi_{R}\left(F_{R}, N_{R}\right)$. It follows that $\pi(F, N) \leq \pi_{R}\left(F_{R}, N_{R}\right)$. Define $P_{R}=F_{R}-c \frac{N_{R}}{Q_{R}}$. Using the fact $F_{R}=C_{P}^{\prime}\left(Q_{R}\right)+\frac{1-\alpha_{1}-\alpha_{2}}{\alpha_{1}} c w_{R}^{t}+c\left(w_{R}^{t}+l\right)-\frac{Q_{R}}{f_{R}^{\prime}}$, we can spell out $P_{R}$ as:

$$
P_{R}=\frac{1-\alpha_{1}-\alpha_{2}}{\alpha_{1}} c w_{R}^{t}-\frac{Q_{R}}{f_{R}^{\prime}}+C_{P}^{\prime}\left(Q_{R}\right)
$$

As $\pi(F, N)$ is assumed to be nonnegative, we have:

$$
\pi_{R}\left(F_{R}, N_{R}\right)=\frac{1-\alpha_{1}-\alpha_{2}}{\alpha_{1}} c w_{R}^{t} Q_{R}-\frac{Q_{R}{ }^{2}}{f_{R}^{\prime}}+Q_{R} C_{P}^{\prime}\left(Q_{R}\right)-C_{P}\left(Q_{R}\right) \geq 0
$$

which leads to:

$$
\frac{1-\alpha_{1}-\alpha_{2}}{\alpha_{1}} c w_{R}^{t}-\frac{Q_{R}}{f_{R}^{\prime}} \geq-C_{P}^{\prime}\left(Q_{R}\right)+\frac{C_{P}\left(Q_{R}\right)}{Q_{R}}
$$

Substituting the above inequality into Eq. (A1), we obtain $P_{R} \geq C_{P}\left(Q_{R}\right) / Q_{R} \geq 0$. Clearly, $F_{R}, N_{R}, P_{R}$ satisfies the constraints of $\mathrm{P} 1$ and thus is feasible. It follows that $\pi_{P}\left(F_{R}, N_{R}, P_{R}\right)=\pi\left(F_{R}, N_{R}\right)=\pi_{R}\left(F_{R}, N_{R}\right)$. Therefore, the optimal solution of the relaxation of $\mathrm{P} 2$ solves $\mathrm{P} 1$.

\section{B. Proof of Proposition 1}

If $\lambda>0$, then $\frac{\partial L}{\partial \lambda_{P}}=\pi_{P}-\pi_{P}^{o}=0$ and $\frac{\partial L}{\partial \lambda_{D}}=\pi_{D}=0$ from the complementary slackness conditions $\lambda_{P} \frac{\partial L}{\partial \lambda_{P}}=0, \lambda_{D} \frac{\partial L}{\partial \lambda_{D}}=0$. If $\lambda=0$, then 
$\pi=\frac{1-\alpha_{1}-\alpha_{2}}{\alpha_{1}} c w^{t} Q+Q C_{P}^{\prime}(Q)-C_{P}(Q)<0$ which conflicts with $\pi \geq \pi_{P}^{o}$. Therefore,

$\pi_{P}=\pi_{P}^{o}, \pi_{D}=0$.

\section{Effects from Customers' Heterogeneous Value of Time}

We can assume a continuous distribution of $\beta$ in the utility functions defined in Eqs. (5)-(6). Generally $F$ and $w^{c}$ in the demand function does not present a linear relationship and thus can be written as:

$$
Q=f\left(F, w^{c}\right)
$$

where $f_{1}<0, f_{2}<0$. Substituting this demand function to all the investigated formulations (P1 P4), one can verify that the tangency condition for the monopoly, firstbest and second-best scenarios are replaced respectively by:

$$
\begin{aligned}
& \frac{f_{1}}{f_{2}}=\frac{1}{c} \frac{\alpha_{1}}{\alpha_{2}} \frac{w^{c}}{w^{t}} \\
& -\frac{Q}{I}=\frac{1}{c} \frac{\alpha_{1}}{\alpha_{2}} \frac{w^{c}}{w^{t}} \\
& \frac{\psi_{1}}{\psi_{2}}=-\frac{f_{2}}{f_{1}} \frac{Q}{I}+\bar{\Delta} f_{2}\left(\frac{f_{2}}{f_{1}} \frac{Q}{I}+1\right)
\end{aligned}
$$

where $I=\int_{F}^{+\infty} f_{2}\left(x, w^{c}\right) d x, \bar{\Delta}=\frac{\partial w^{c}}{\partial Q}, \psi_{1}=F-C_{P}{ }^{\prime}(Q)+c /\left(\frac{\partial Q}{\partial N}\right)$ and $\psi_{2}=F-C_{P}{ }^{\prime}(Q)+Q /\left(\frac{\partial Q}{\partial F}\right)$. Note when calculating the consumers' surplus, we fix the average waiting time at the equilibrium level and integrate under a hypothetical market demand curve (Cairns and Liston-Heyes, 1996; Yang et al., 2002).

If $f_{2}=\beta f_{1}$ (the case of homogenous value of waiting time), Eqs. (A5)-(A7) will reduce to the tangency condition.

\section{Sensitivity Analysis}

Expressing the Cob-Douglas type matching function utilizing the tangency condition, we have: 


$$
Q=A Q^{\alpha_{1}+\alpha_{2}}\left(w^{t}\right)^{\alpha_{1}}\left(\frac{c}{\beta} \frac{\alpha_{2}}{\alpha_{1}} w^{t}\right)^{\alpha_{2}}=A^{-\left(1-\alpha_{1}-\alpha_{2}\right)} \xi_{2}\left(w^{t}\right)^{\frac{\alpha_{1}+\alpha_{2}}{1-\alpha_{1}-\alpha_{2}}}
$$

where $\xi_{2}=\left(\frac{c}{\beta} \frac{\alpha_{2}}{\alpha_{1}} w^{t}\right)^{\frac{\alpha_{2}}{1-\alpha_{1}-\alpha_{2}}}$. Further, at equilibrium,

$$
\theta \mu+\ln Q=\theta C+\ln (\bar{Q}-Q)
$$

Without loss of generosity, we assume the dispersion parameter to be 1 . Differentiating the above equations w.r.t $A$ :

$$
\begin{aligned}
& \frac{\partial Q}{\partial A}=\frac{1}{1-\alpha_{1}-\alpha_{2}} \frac{Q}{A}+\frac{\alpha_{1}+\alpha_{2}}{1-\alpha_{1}-\alpha_{2}} \frac{Q}{w^{t}} \frac{\partial w^{t}}{\partial A} \\
& \frac{\partial \mu}{\partial A}=-\frac{\bar{Q}}{Q(\bar{Q}-Q)} \frac{\partial Q}{\partial A}
\end{aligned}
$$

The specification of the generalized cost $\mu$ depends on the investigated scenario.

Case (1): Zero-profit second-best.

$\mu=c\left(w^{t}+l\right)+\bar{C}_{P}+c \frac{\alpha_{2}}{\alpha_{1}} w^{t}+\tau l$. Together with Eqs. (A10) and (A11):

$$
\left(1+\frac{\alpha_{2}}{\alpha_{1}}\right) c \frac{\partial w^{t}}{\partial A}=-\frac{\bar{Q}}{Q(\bar{Q}-Q)}\left(\frac{1}{1-\alpha_{1}-\alpha_{2}} \frac{Q}{A}+\frac{\alpha_{1}+\alpha_{2}}{1-\alpha_{1}-\alpha_{2}} \frac{Q}{w^{t}} \frac{\partial w^{t}}{\partial A}\right)
$$

Re-arranging the terms, we obtain:

$$
\frac{\partial w^{t}}{\partial A}=\frac{-w^{t} \frac{1}{\alpha_{1}+\alpha_{2}} \frac{\bar{Q}}{A(\bar{Q}-Q)}}{\frac{1-\alpha_{1}-\alpha_{2}}{\alpha_{1}} c w^{t}+\frac{\bar{Q}}{(\bar{Q}-Q)}}
$$

It is straightforward to see that the numerator is negative. Next, we will show the sign of the denominator is positive. Given the assumption that the joint profit for the monopoly solution is nonnegative: 


$$
F^{*} Q^{*}-c N^{*}-C_{P}\left(Q^{*}\right)=Q\left(\frac{1-\alpha_{1}-\alpha_{2}}{\alpha_{1}} c w^{t *}-\frac{Q^{*}}{f^{* \prime}}\right) \geq 0
$$

The asterisk denotes the monopoly solution. For the specified demand function

$$
\begin{gathered}
\frac{Q^{*}}{f^{* \prime}}=-\frac{\bar{Q}}{\bar{Q}-Q^{*}} \text {. Therefore we have: } \\
\frac{1-\alpha_{1}-\alpha_{2}}{\alpha_{1}} c w^{t *} \geq-\frac{\bar{Q}}{\bar{Q}-Q^{*}}
\end{gathered}
$$

When the demand increases from the monopoly to the second-best level, $w^{t}<w^{t *}, Q>Q^{*}$. Therefore, at the second-best:

$$
\frac{1-\alpha_{1}-\alpha_{2}}{\alpha_{1}} c w^{t}>\frac{1-\alpha_{1}-\alpha_{2}}{\alpha_{1}} c w^{t *} \geq-\frac{\bar{Q}}{\bar{Q}-Q^{*}}>-\frac{\bar{Q}}{\bar{Q}-Q}
$$

That is, $\frac{1-\alpha_{1}-\alpha_{2}}{\alpha_{1}} c w^{t}+\frac{\bar{Q}}{(\bar{Q}-Q)}>0$ which gives $\frac{\partial w^{t}}{\partial A}<0$ at the second-best. This result still holds for other second-best solutions with varying reservation profit levels.

Case (2) Monopoly solution $\mu^{*}=c\left(w^{t *}+l\right)+\bar{C}_{P}+\frac{1-\alpha_{1}-\alpha_{2}}{\alpha_{1}} c w^{t *}+\frac{\bar{Q}}{\left(\bar{Q}-Q^{*}\right)}+c \frac{\alpha_{2}}{\alpha_{1}} w^{t *}+\tau l$. Then Eq. (A11) can be rewritten as:

$$
\frac{\partial w^{t *}}{\partial A}=-\frac{\alpha_{1}}{c} \frac{\bar{Q}^{2}}{Q\left(\bar{Q}-Q^{*}\right)^{2}} \frac{\partial Q^{*}}{\partial A}
$$

Substituting $\frac{\partial Q^{*}}{\partial A}$ as specified in Eq. (A10) into Eq. (A17) leads to:

$$
\frac{\partial w^{t *}}{\partial A}=\frac{-\frac{1}{\left(1-\alpha_{1}-\alpha_{2}\right)} \frac{1}{A}}{\frac{c}{\alpha_{1}} \frac{\left(\bar{Q}-Q^{*}\right)^{2}}{\bar{Q}^{2}}+\frac{\alpha_{1}+\alpha_{2}}{1-\alpha_{1}-\alpha_{2}} \frac{1}{w^{t *}}}
$$


The numerator of $\frac{\partial w^{t *}}{\partial A}$ is positive. From Eq. (A15):

$$
\frac{1}{w^{t *}} \geq c \frac{\alpha_{1}+\alpha_{2}-1}{\alpha_{1}} \frac{\bar{Q}-Q^{*}}{\bar{Q}}
$$

Then

$$
\frac{c}{\alpha_{1}} \frac{\left(\bar{Q}-Q^{*}\right)^{2}}{\bar{Q}^{2}}+\frac{\alpha_{1}+\alpha_{2}}{1-\alpha_{1}-\alpha_{2}} \frac{1}{w^{t *}} \leq \frac{c}{\alpha_{1}} \frac{\bar{Q}-Q^{*}}{\bar{Q}}\left(\frac{\bar{Q}-Q^{*}}{\bar{Q}}-\left(\alpha_{1}+\alpha_{2}\right)\right)<0(\mathrm{~A} 20)
$$

which indicates $\frac{\partial w^{t *}}{\partial A}<0$ at the monopoly solution. Following a similar procedure, one can verify the sign of $F, Q$. 